\title{
El ejercicio de la profesión contable en Colombia. Una mirada desde el paradigma contable del comportamiento basado en la conducta y el deterioro de la salud física y mental
}

\author{
The practice of accounting in Colombia: A look to conduct-based behavior and physical \\ and mental health deterioration
}

Recibido: 04/09/13 - Aprobado versión final: 03/11/14

Edilgardo Loaiza Betancur*

\begin{abstract}
Resumen: el ejercicio de la profesión contable en Colombia se ha observado bajo ciertas condiciones laborales, como una profesión que genera impacto en la salud física y mental del profesional. Se presenta como avance de investigación, una reflexión que se enmarca dentro de la Investigación Contable del Comportamiento - ICC - por medio de un contraste entre antecedentes de investigaciones realizadas y literatura especializada en ciencias del comportamiento. Se evidenciaron coincidencias entre resultados de investigaciones realizadas y la caracterización tipológica del profesional contable, abordada desde la psicología laboral y la sociología de las profesiones. Se destacan coincidencias entre las diferentes investigaciones sobre la inmensa carga emocional que debe soportar este profesional, lo que afecta su salud y sus relaciones sociales y afectivas, y constituye un costo psicosocial y psicoafectivo importante que debe asumir para alcanzar el éxito profesional.
\end{abstract}

Palabras clave: estrés en contadores, personalidad del contador, comportamiento contable, profesión contable, burnout en contadores.

\begin{abstract}
Colombia has been deemed, under certain work conditions, a profession that generates an impact in the practitioner's physical and mental health. This reflection is presented as an advance of research, in the framework of the research project Behavior Research in Accounting (ICC). It contrasted previous research background specialized literature on the science of behavior. The findings of previous research works and the typological characterization of accounting practitioners performed by work psychology and the sociology of professions showed coincidences. Worth of note are coincidences among the various reserch works on the huge emotional burden these practitioners are subject to, affecting their health and their social and affective relationships, and constituting a major psycho-social and psycho-affective burden, which they need to cope with in order to achieve professional success.
\end{abstract}

Keywords: stress among accounting practitioners, accountant's personality, accounting behavior, accounting profession, burnout among accounting practitioners.

\section{JEL: I31 - M54}

* Doctor en Ciencias Contables, Especialista en Métodos Administrativos, Contador Público, Administrador en Salud y docente del Politécnico Colombiano Jaime Isaza Cadavid, Medellín - Colombia. eloaiza@elpoli.edu.co 


\section{Contexto Loaiza, E.}

\section{La profession d'expert-comptable en Colombie: une approche sur le paradigme comptable du comportement à propos de la détérioration de la santé physique et mentale du professionnel}

Résumé: du point de vue des conditions de travail, il s'est avéré que l'exercice de la profession d'expert-comptable en Colombie n'est pas sans conséquences sur la santé physique et mentale des professionnels. Ici il est question de présenter les premiers résultats d'une recherche effectué à partir d'une approche comportementale. Il s'agit donc d'une Recherche Comptable du Comportement-RCC-. Il a été question de réaliser un contraste entre des recherches préalables et la littérature spécialisée des sciences du comportement. Des coïncidences ont été trouvées entre les résultats des recherches préalables et la caractérisation de l'expert-comptable faite par la psychologie du travail et la sociologie des professions. Ces coïncidences portent sur l'énorme stress que doit subir l'expert-comptable et ses conséquences sur la santé et la vie sociale et personnelle de ce dernier ; il en découle un important coût psychosocial et psychoaffectif que le professionnel doit assumer afin de réussir professionnellement.

Mots clé : stress chez les experts-comptables, personnalité de l'expert-comptable, métier de l'expert-comptable, burnout chez les experts-comptables.

\section{Introducción}

El ejercicio de la profesión contable en Colombia bajo determinadas condiciones extremas de carga física y mental y su efecto en la salud es un fenómeno estudiado desde décadas atrás. Se presenta este artículo como resultado parcial de investigación de una tesis doctoral que apunta a medir el fenómeno desde una ecuación que integra variables y categorías (Loaiza, 2014). Este artículo tiene como objetivo realizar un contraste entre los antecedentes de investigaciones realizadas sobre la problemática y literatura especializada que busca caracterizar la personalidad del contador público desde la psicología laboral y la sociología de la profesión contable. Sea entonces una reflexión crítica, frente al ejercicio de la profesión contable y su impacto en la salud, abordándolo como fenómeno desde el paradigma contable del comportamiento basado en la conducta y en el que el contable intersubjetivamente se observa dentro del ejercicio profesional, trascendiendo lo estrictamente operativo, para insertarse en ámbitos complejos del comportamiento humano, por eso se aborda desde ciencias del comportamiento como la sociología y la psicología, aplicándolas a la administración, la economía y la contabilidad con un enfoque de gestión del talento humano y hacia líneas investigativas de responsabilidad social del profesional, de la empresa y del Estado, y la línea de investigación contable basada en la conducta con un método cualitativo fenomenológico tendiente a interpretar y dar explicación al fenómeno. 


\section{Antecedentes}

Esta investigación inició con una observación metódica del fenómeno, que el autor abordó mediante sondeos de opinión y de revisión bibliográfica sobre estrés y síndrome de burnout en profesionales, pero con pocos resultados en el caso de contadores públicos en el ámbito nacional. Sin embargo, despertó interés un registro documental de gran rigurosidad metodológica relacionado con un estudio sobre riesgos psicosociales en personas que laboran en áreas administrativas en la ciudad de Medellín. (Montoya \& Posada, 2007)

La investigación destaca a los contadores públicos, entre otros profesionales, como "trabajadores que tienden a sufrir de marcados síntomas y dolencias por las características y condiciones de sus trabajos, que no sólo causan ausentismo laboral sino que también dañan, en forma lenta y progresiva, la salud física y mental", y menciona, entre otros trastornos:

[...] el de columna, por las posiciones poco ergonómicas, las tendinitis y tenosinovitis, que son la expresión de los movimientos derivados del uso del mouse, irritabilidad de ojos como consecuencia del uso permanente del computador, lo que en ocasiones disminuye la visión, síndrome del ojo seco dado por la falta o disminución del parpadeo, entre otros síntomas y afecciones. (Montoya \& Posada, 2007, p. 15-18)

En el ámbito latinoamericano los resultados de la revisión documental no fueron los más contundentes. Sin embargo, las psicoanalistas C. Arredondo y A. Villavicencio, en el año 2007 expusieron a la comunidad científica resultados de investigación sobre el riesgo psicosocial de estudiantes de contaduría pública (Arredondo \& Villavicencio, 2007). En su estudio exploratorio a 107 estudiantes, se obtuvo que el $70 \%$ presentaban inestabilidad psicológica y pudieran estar en una fase crítica para afrontar el estrés (Villavicencio, 2006). Si bien el enfoque holístico y las escalas e instrumentos psicométricos utilizados en este estudio se alejan un poco del estrés generado por el desempeño profesional del contador, ya evidencia interés de profesionales de otras disciplinas por las prácticas laborales del profesional contable desde su proceso de formación.

Los resultados de la búsqueda dejaron sentado que la problemática se viene estudiando desde hace más de cuarenta años, principalmente en Estados Unidos, debido a que en este país, y como consecuencia del estilo de trabajo que caracteriza a las grandes multinacionales de auditoría, la productividad por estándares y la presión del tiempo para la entrega de informes tributarios y de auditoría abruman a estos profesionales.

La revisión documental arrojó que el fenómeno del impacto en la salud mental del contable como consecuencia del trabajo extremo, ha generado avances desde una línea de investigación contable del comportamiento 


\section{Contexto Loaiza, E.}

basado en la conducta, muy explorada y estudiada pero poco difundida en la literatura contable especializada en el ámbito nacional. Estas circunstancias que históricamente se están evidenciando, han ofrecido pautas a diferentes investigadores contables y a otras ciencias y disciplinas para categorizar aspectos psicológicos y sociológicos que progresivamente han contribuido a un nuevo conocimiento contable, relacionado con la interrelación entre el trabajo, el ambiente, la sociedad, el estado y la subjetividad del individuo. Ello, sin lugar a dudas, confluye con el desarrollo epistemológico y teleológico de la contabilidad.

Se ha tratado, entonces, al sujeto como objeto de estudio desde la psicología organizacional y del trabajo, con aportes desde la teoría contable y administrativa y la sociología del trabajo, enfoques profundamente humanísticos en pro de la mejora de la calidad de vida de los contables del mundo.

Temas como el estrés de rol con sus dimensiones de ambigüedad, conflicto y sobrecarga de rol, que han sido estudiados por algunos autores (Hellriegel \& White, 1973; Senatra, 1980; Rebele \& Michaels, 1990; Almer \& Kaplan, 2002; Jones, 2007), evidencian la relación entre la salud mental del contable y los procesos cada vez más complejos de la contabilidad, la auditoría y los impuestos, en ambientes disciplinares diversos en los cuales debe desempeñarse este profesional, y que en muchas ocasiones desborda la capacidad de respuesta con la consecuente presencia de altos niveles de estrés. Aspectos asociados con la actitud del individuo y con la tipología de la personalidad explican las conductas con altas relaciones disfuncionales en salud mental, como en los casos de aquellos individuos con personalidad tipo A, los cuales son propensos a desarrollar niveles elevados de estrés, dado su carácter obsesivo, argumento fundamentado en algunas investigaciones. (Haskins, Baglioni, \& Cooper, 1990; Fogarty et al., 2000; Fisher, 2001; Donnelly, Quirin, \& O’Bryan, 2003; Ciftcioglu, 2011; Loaiza, 2012)

Estos rasgos y tipos de personalidad que desarrollan ciertas conductas han sido estudiados también desde las habilidades del individuo, lo que se denomina locus de control (el sentido de control a la causa de su experiencia o forma de vida), el cual se compone de locus de control interno (la causa del éxito o fracaso es ubicada o percibida por la persona como algo interno a ella) y el locus de control externo (persona percibe que los resultados dependen de causas o factores externos, como pueden ser la suerte y el destino). Por otro lado, un clima organizacional competitivo, con estilos administrativos basados en las metas y estándares cada vez más exigentes y con jefes arrogantes o tóxicos, generan altos niveles de estrés y el efecto es totalmente contrario, tal como se ha demostrado. (Hellriegel \& White, 1973; Donnelly et al., 2003; Ussahawanitchakit, 2008; Cadogan-McClean, 2009; Piñuel, 2004) 
Es importante destacar que el Estado tiene un papel importante en esta problemática, en el sentido de que toda política monetaria involucra una política fiscal, y cualquier reforma puede incrementar la carga laboral en los profesionales contables, tal como se evidenció en Estados Unidos (1986), cuando al darse la reforma tributaria se incrementó considerablemente la carga laboral de los contables, sobre todo en el primer semestre del año. Si bien esos estudios se efectuaron en este país, en Australia también se presentó un efecto similar (Kalbers \& Fogarty, 2005; Phillips \& De Lange, 2006). Es casi una lógica que conduce a afirmar que ocurre lo mismo en los demás países, entre ellos Colombia.

El impacto desde la perspectiva de la psicología del trabajo lo acometen Lovelace, Manz, \& Alves (2007), cuando exponen que las tendencias actuales de trabajo incluyen más horas diarias de dedicación, aumentando las demandas y presiones de gestión dentro de la competencia, lo que genera inseguridad laboral y la pérdida de control sobre el trabajo, y se convierte en preocupación importante del profesional contable.

\section{Metodología}

Como resultado parcial de investigación, este escrito propone una reflexión crítica frente al ejercicio de la profesión contable, su impacto en la salud física y mental, y la necesidad de profundizar en el desarrollo de investigaciones que apunten a la explicación del fenómeno.

La motivación intersubjetiva del autor hace pensar que se estudia a sí mismo, lo que no necesariamente se puede asumir como sesgo, dado que desde el concepto de epistemetodología, abordado por Viloria (2001, p. 64), el "método" para construir conocimiento se integra íntimamente con el modo de obtenerlo, y el propio investigador se convierte, a su vez, en investigado por las ciencias sociales, puesto que "las relaciones internaexterna, individual-social, cognitivo-afectiva, están presentes en el hombre y afloran en todas sus acciones".

El método mediante el cual se pretende dar respuesta a las inquietudes surgidas de esta investigación está asociado con la comprensión del fenómeno, de acuerdo con las vivencias de los sujetos contables, de tal forma que éstos no solo contemplan el "mundo", también se ven a sí mismos como testigos de sus propias experiencias. Igualmente, la gran carga asociada al comportamiento del contable desde la perspectiva de la salud, hace que se estudie no solo al contable como persona sino también a su entorno.

Como expresión directa de la filosofía fenomenológica expuesta por Edmund Husserl (1859-1938), el método fenomenológico aborda la realidad dentro del fuero interno del individuo para tratar de explicarla. A partir de Husserl se enfatiza en la intuición reflexiva para describir la experiencia tal como se 


\section{Contexto Loaiza, E.}

vive y, desde su punto de vista, todas las distinciones de nuestra experiencia deben carecer de presuposiciones y prejuicios; sin embargo, se reclama buscar los fundamentos teóricos que permitan crear una base segura para describir la experiencia y conseguir la realidad del mundo tal y como es. (Trejo, 2012)

El enfoque utilizado permite identificar su temática a partir de la contabilidad de gestión y la investigación contable del comportamiento, y no de la perspectiva del paradigma de la utilidad de la información contable ni del comportamiento de las cifras, y la influencia en la gerencia para la toma de decisiones sino con base en la conducta del contable y su influencia en la calidad de los informes.

En este sentido, técnicas de investigación contable como la observación participante y la revisión documental, utilizadas en esta investigación son estrategias sociales-cualitativas acogidas por la investigación heterodoxa, sin embargo, la investigación heterodoxa no se limita a estrategias exclusivamente cualitativas. La corriente crítica, por ejemplo, aboga por el uso de metodologías tanto cualitativas como cuantitativas, por eso, si este fenómeno se abordara con la epidemiología, por ejemplo, se obtendrían inferencias que lo analizan y explican, no obstante, el enfoque meramente positivista de la estadística puede migrar a un enfoque emergente, el cual se fundamenta en la fenomenología de Edmund Husserl, que captura la esencia de los fenómenos y de la vida humana desde la intersubjetividad y la subjetividad.

Por su parte, Galindo (1994) manifiesta que:

[...] el sujeto investigador tiene un impulso hacia el objeto por configurar que reclama todo su ser, toda su posibilidad. Este movimiento tiene un largo momento interior que se proyecta hacia afuera en un programa de acción y sentido que busca entender más, ampliar el horizonte del objeto haciendo crecer al sujeto. (p. 9)

Es poco posible comprender como fenómeno los comportamientos y las conductas por separado, mediante los métodos tradicionales de investigación, en un contexto determinado y bajo condiciones afectadas por los sentimientos y las pasiones que generan unos resultados producto de unas interacciones.

\section{Resultados}

\section{Consideraciones sobre el desempeño de la profesión contable en Colombia}

La profesión del contador público está regulada mediante la Ley 43 de 1990, bajo la cual, para efectos legales, se entiende por contador público: 
La persona natural que, mediante la inscripción que acredite su competencia profesional en los términos de la ley, está facultada para dar fe pública de hechos propios del ámbito de su profesión, dictaminar sobre estados financieros, realizar las demás actividades relacionadas con la ciencia contable en general [...].

Se enuncia esta definición para resaltar que el contador público otorga fe pública sobre los actos del administrador en una empresa privada, y este elemento le endilga una alta responsabilidad de tipo social, la cual se puede convertir en una categoría de presión con relación de proporcionalidad en el nivel axiológico y ético del contador, quien no escapa a los efectos que la norma comercial, penal, administrativa y de régimen disciplinario que ejerce la Junta Central de Contadores, determine sobre sus actos. ${ }^{1}$

De la misma norma se extrae lo siguiente:

[el contador público] puede desempeñar las funciones de revisor fiscal, auditor externo, auditor interno en toda clase de sociedades; también puede ser jefe de contabilidad o su equivalente, visitador estatal en asuntos técnico-contables, representando autoridades gubernamentales de tributación, vigilancia y control. Asimismo, el Contador Público puede actuar como perito en controversias de carácter técnico-contable, especialmente en diligencias sobre exhibición de libros, juicios de rendición de cuentas, avalúo de intangibles patrimoniales y costo de empresas en marcha.

La precitada norma fue publicada hace más de dos décadas, y con el desarrollo de los sistemas de información y la tecnología para acceder a ella, se observa que los modelos se han desarrollado y los roles también, y en la actualidad el contador público tiene un amplio espectro de funciones y actividades, que van desde el simple registro de transacciones hasta el mismo diseño de sistemas de información, lo cual salta del ámbito estrictamente contable a otros ámbitos como el administrativo, económico, financiero, productivo, de calidad, medio ambiente y normatividad internacional, entre otros; de acuerdo con el tipo de empresa en la que se labore. Por lo tanto, el rol inicial del contador público según la norma, dista mucho de la práctica actual, ya que si en su esencia es la misma, en la forma se ha complejizado y hasta diluido entre otras actividades propias de otras profesiones, lo que ha generado una disfunción del rol laboral.

En el ámbito estatal la Contaduría General de la Nación tuvo su origen en la Asamblea Nacional Constituyente de 1991, y se creó la figura del Contador General de la Nación (Artículo 354), y se dispuso que "habrá un Contador General, funcionario de la rama ejecutiva, quien llevará la

1. La Junta Central de Contadores Públicos de Colombia es un organismo estatal responsable de llevar a cabo el registro y control de personas naturales y jurídicas prestadoras de servicios contables, y actúa además como ente de control disciplinario. 


\section{Contexto Laiza, E.}

contabilidad general de la nación y consolidará ésta con las de sus entidades descentralizadas". Estas funciones deben ser realizadas por un contador público, y se rigen por estrictas normas del orden contable y administrativo cuyo control también está reglamentado en la misma Constitución (Artículo 267), mediante la figura de la Contraloría General de la Nación con sus diferentes niveles del orden territorial, (departamentos y municipios), que también requieren de un contador público para el desempeño de sus funciones.

Este aspecto de la vida nacional demanda la participación de contadores públicos con altas cuotas de responsabilidad y compromiso, unido a que la sociedad ejerce presión sobre la confianza que brinda el contador con su firma, debido a los actos de corrupción que se han presentado y evidenciado en los últimos años en entidades estatales y privadas, y sobre los cuales dicha sociedad se ha preguntado: ¿Y dónde estaban los contadores, revisores fiscales, auditores o contralores? ¿Por qué no alertaron o revelaron o denunciaron tantas irregularidades?

El Gobierno colombiano institucionalizó la figura de Revisoría Fiscal con la Ley 73 de 1935, y luego, motivado por el desarrollo industrial y comercial,y la imperante necesidad de establecer controles de orden fiscal asignó al contador público como profesional idóneo para ejercer dicha responsabilidad, lo que se convirtó en un imperativo categórico mediante el Decreto 2373 de 1956. En la actualidad, esta figura controla el manejo de las empresas que estén obligadas a tenerla, vela por el cumplimiento de los deberes legales y estatutarios de las mismas, y verifica si los estados financieros reflejan fielmente la situación financiera de la entidad y sus resultados económicos. Estas responsabilidades también están reglamentadas en el Código de Comercio, mediante el Decreto 410 de 1971, y se complementan con la Ley 222 de 1995.

Pero la figura de revisor fiscal va más allá de la norma y enmarca aspectos de tipo ético y axiológico propios de la responsabilidad social profesional, y en este aspecto la labor del revisor fiscal, fuera de estar, en ocasiones cuestionada, en detrimento de su imagen y existencia como figura, tiene que propender por restituir una confianza pública que se ha menoscabado a raíz de escándalos de tipo económico y financiero, que ya no generan asombro por su frecuencia. Estos niveles de responsabilidad pudieran ser considerados como factores de riesgo psicosocial externo, debido a que no sólo existe la exigencia social que le demanda otorgar fe pública, sino un régimen sancionatorio para aquellos contadores que no alerten oportunamente, en sus respectivos informes, sobre situaciones de alto riesgo financiero para la empresa, el Estado o la sociedad.

Ahora bien, un elemento que se debe considerar como un factor externo es el control que se pretende realizar desde la macroeconomía de los países, el cual ya lo manifestaba la colega Aída Calvo al argumentar que: 
En el proceso de análisis de las dinámicas externas a la profesión contable se hace necesario considerar las transformaciones de los ámbitos regulativos tanto económicos como jurídicos, ya que a través de éstos se develan y analizan las relaciones de control y poder que influyen en su respectiva configuración, lo cual se puede interpretar a la luz de los cambios en las orientaciones regulativas de la profesión Contable en Colombia. (2007, p. 82)

Estos cambios a los que se refiere Calvo se materializan hoy en las discusiones sobre convergencia de las Normas Internacionales de Contabilidad, que si bien se dan como un hecho, existen cuestionamientos de forma y fondo en su fin último, situación que aún genera controversia de tipo gremial.

Si a todo lo anterior se suma el hecho de que la legislación tributaria colombiana se aleja cada vez más de las normas contables, y se agrega una conciliación tributaria que cada vez se hace más compleja, y sobre la que también el contador recibe presión del administrador o dueño para optimizar el recurso financiero destinado al pago de cargas tributarias, y que cada vez hay mayor presión de las autoridades tributarias para aplicar desde la exégesis dicha norma, con lo que el profesional contable pierde gradualmente independencia mental, lo que constituye uno de los principios rectores de su labor diaria.

Se destaca además que pocas veces se puede hacer interpretación jurídica o hermenéutica, por el régimen de sanciones pecuniarias a que está expuesto este profesional por incumplimiento en las fechas de presentación de declaraciones tributarias o por no usar protocolos o formatos contables, lo que puede invalidar la información presentada o por no aplicar normas y principios contables, todo lo cual conduce a frenéticas carreras en épocas de "vencimientos" que en ocasiones abruman al contador y a todo su equipo de trabajo.

Al panorama nacional se suma que en algunas empresas multinacionales la información financiera se debe convertir a formatos y esquemas propios de sus casas matrices, las cuales exigen la presentación de reportes financieros bajo normas internacionales, lo que obliga al contador a re-expresar lo que ya había realizado para cumplir con la exigencia contable y tributaria de Colombia.

En el campo organizacional se presenta a menudo una disfunción de rol, porque el contador público se involucra sistémicamente en procesos que se relacionan con su saber específico pero que no forman parte de sus responsabilidades como profesional, y participa entonces en diversos comités primarios y otras actividades que le demandan tiempo adicional en detrimento de sus responsabilidades de tipo contable. La dinámica administrativa exige calidad y oportunidad en la información, la cual debe ser preparada en ocasiones con pocos recursos humanos o inadecuados recursos tecnológicos (software y hardware), que en algunas organizaciones son limitados no solo por el recurso financiero escaso sino por la poca 


\section{Contexto Laiza, E.}

importancia estratégica que a veces se le concede a la información contable, ya que su uso marca un enfoque predominantemente tributario.

Desde la sociología de las profesiones nuevamente se cita a la colega Aída Calvo, quien afirma que "las dinámicas que se están dando en el ámbito de la profesión contable deben interpretarse desde una óptica interdisciplinaria, con el fin de que se alcance la comprensión de los diversos actores implícitos y explícitos en los distintos planos de interacción" (Calvo, 2007, p. 79). Por eso este estudio compromete otros saberes para poder abordar, desde una perspectiva más amplia, el ejercicio de la profesión contable como fenómeno.

Aspectos como la independencia mental, la fe pública y la responsabilidad social dentro de contextos regionales, nacionales e internacionales pueden ser precursores de un fenómeno que hace cada vez más complejo el ejercicio de la profesión contable, y que analizados con base en seis grupos de factores, propuestos como determinantes de la salud, se podrían establecer etiologías del deterioro de la salud física y mental del contador público en ejercicio de su profesión.

Estos seis factores se presentan en forma sistémica de lo general a lo particular, siendo cada uno de ellos un universo en sí mismo: factores políticos y económicos (Neoliberalismo y mercado globalizado); factores ambientales (urbanismo y condiciones de vida); factores sociales (convivencia, industrialización, nuevas tecnologías y mercado); factores organizacionales (estilos administrativos y organización del trabajo); factores laborales (exigencias ocupacionales intrínsecas), y factores individuales (personalidad y comportamiento). Este último grupo de factores, los individuales, dan entrada al abordaje de este escrito como incursión a una caracterización de la profesión contable desde la salud mental y el trabajo, en concordancia con su propia interacción estudiada a partir de la sociología de las profesiones.

\section{Contabilidad y comportamiento, una imbricación inexorable}

La sociología y la psicología, como ciencias del comportamiento, se pueden aplicar en la administración, la economía y la contabilidad. Honrgren (1962, citado en Birnberg, 2000) manifestó desde hace más de cinco décadas que el comportamiento humano también ha sido tema de investigación como influencia en los sistemas de información contable y en la determinación de su eficacia. Birnberg (2000) menciona algunos estudios centrados en indicadores, incentivos, estándares y presupuestos, y su influencia en el comportamiento y la conducta de los contables y personal administrativo; y destaca, por ejemplo, que muchos de los comportamientos observados en algunas empresas son respuestas inadecuadas a los datos contables, y se consideran disfuncionales u oportunistas debido a presiones por rendimiento y cumplimiento de metas y propósitos de obligatorio cumplimiento. 
Los planteamientos de Honrgren (1962) se siguen desarrollando, y ocho años más tarde se conocen once enfoques de Hendriksen (1970) elevados bajo su criterio a paradigmas de la contabilidad, uno de los cuales lo denominó "enfoque basado en la conducta". Hoy en día se siguen tomando estos conceptos para el desarrollo de la temática como línea de investigación. Montes, Soto y Valencia, en su escrito denominado "Paradigmas de la contabilidad", retoman a Hendriksen y argumentan:

Los estudios del comportamiento analizados por la psicología, la sociología y la economía, los puede estudiar y analizar la contabilidad, también incluirlos en su teoría para predecir y determinar los comportamientos económicos de los transactores del mercado. La contabilidad puede entenderse entonces como una variable que influye en el comportamiento de las personas naturales y jurídicas en la toma de decisiones individuales y colectivas. (2006, p. 64)

\section{Conductas y comportamientos del profesional contable con riesgos psicosociales y psicoafectivos}

Al revisar la información bibliográfica fundamento del presente estudio, se encuentra un artículo en la Revista Semana (2012), según el cual en una consulta que realizó la empresa multinacional Manpower, especializada en reclutamiento de personal, a 39.000 empleadores de todo el mundo sobre la escasez del talento humano y su afectación en la organización, se encontró que "tres de cada diez empresarios reconocieron tener dificultades para cubrir puestos de trabajo", y que en el caso colombiano, el personal contable y financiero ocupó el primer lugar de los diez perfiles más críticos para conseguir por falta de talento disponible, esto debido a que las empresas "buscan personas con especialidades muy concretas, por ejemplo, en contabilidad global, expertos en normas internacionales, en reestructuraciones de deudas o en ciertas auditorías, lo que dificulta la tarea de reclutamiento".

Lo anterior se podría explicar, en parte, por el desarrollo y la diversificación de las transacciones económicas, así como por la modernización del mercado en un contexto global. De ahí que la demanda de información financiera y contable pretenda cumplir objetivos cada vez más diversos, que conducen a nuevas formas de concebir y comprender los sistemas de información, desde una perspectiva del trabajo como fenómeno psicosocial. Peiró y Prieto (2002) ya se anticipaban a esta situación, cuando argumentaban que "[...] los trabajos requieren cada vez una mayor capacidad de elaboración y representación mental, de las situaciones y procesamientos de la información, lo que requiere también transformaciones importantes en la formación y en la preparación de las personas" (p. 27). Y más adelante plantean: "la interacción entre la persona y el entorno del trabajo y su desempeño (p. ej., rendimiento laboral), determina también los productos y resultados relacionados con la tarea, en términos de eficacia, calidad, velocidad y precisión" (p. 29). En el 


\section{Contexto losia}

caso del desempeño laboral del contador público en Colombia, la velocidad y precisión son un imperativo, y la eficacia y calidad están implícitas, ya que se rigen por normas y reglamentos; por lo tanto, la pericia en este caso se debe desarrollar con anterioridad.

¿Pero qué tiene que ver esto con algunos comportamientos observados en contadores públicos? Algunas conductas y comportamientos que estudian la psicología y la sociología coinciden con actitudes del profesional contable que sugieren la presencia de riesgos psicosociales y psicoafectivos. Se propone una relación entre el tipo de trabajo y algunos comportamientos del individuo como fuente de desarrollo de algunos rasgos de personalidad. Esto se puede reforzar con el planteamiento según el cual el trabajo es una fuente de identidad personal, ya que según Peiró y Prieto (2002), "el trabajo es una de las áreas de mayor importancia para el desarrollo y la formación de nuestra identidad. Cómo somos y cómo nos vemos, tiene mucho que ver con cómo somos, cómo nos vemos y cómo nos ven en el trabajo" (p. 40). Estas conductas o comportamientos aprendidos son propios de sociedades altamente competitivas, como las promovidas por el consumismo, la sobreproducción y la acumulación desmedida de riqueza material y de poder, propias del "capitalismo salvaje", término acuñado a partir del neoliberalismo económico.

Piñuel (2004) caracteriza al contador público entre las "personalidades obsesivo compulsivas", y menciona, por ejemplo, que "el perfeccionismo está claramente implicado en algunos tipos de adicción al trabajo. El hecho de tener rasgos de personalidad que comúnmente denominamos 'perfeccionistas', suele desempeñar también un papel precursor del workaholismo" (p. 151). Este perfeccionismo se asocia con el contador público como una de sus características "workaholicas", que significa adicción al trabajo, cuyos rasgos los relaciona Piñuel con: "el perfeccionismo, la obstinación (terquedad), la reiteración (repetición de comportamientos), la perseverancia (tenacidad), la laboriosidad, la meticulosidad (la atención a los detalles), el coleccionismo, la rigidez y la intolerancia a la ambigüedad". Estos rasgos del comportamiento no se alejan de la realidad cuando se observa en detalle al contador público en pleno ejercicio de su profesión.

Argumenta también este autor que las personas con estos rasgos de personalidad tienden a seleccionar "puestos de trabajo que requieren atención a los detalles, controlar, chequear datos, repetir meticulosamente tareas, observar detalladamente reglas, procedimientos o consignas, cuadrar números" (Piñuel, 2004, p. 152). Dentro del listado de profesiones y cargos relacionados con el ejercicio de la contaduría pública con alta prevalencia de individuos con este tipo de personalidad, se encuentran los "controllers", administradores, financieros, contables, analistas y programadores.

Este tipo de personalidades - continúa Piñuel - "buscan desarrollarse intelectualmente hasta alcanzar la omnisciencia (saber todo de todo)", y muestra con un nivel de detalle asombroso lo que se podría inferir como 
los rasgos más comunes de un contador público, cuando argumenta que "la necesidad de saberlo todo de todo y el perfeccionismo compulsivo generan falta de disposición para asumir riesgos" (p. 153). Este perfeccionismo, característico de este tipo de personalidad, lo aleja de la toma de decisiones que implican asumir riesgos, para eliminar la posibilidad de cometer errores. A lo mejor esto explica por qué al autor de este estudio le costó quince años de su vida antes de que fuera despedido de "su" empresa, para darse cuenta del fenómeno que estaba viviendo.

Por otra parte, un patrón de personalidad que se asocia con algunas variables del síndrome de burnout, y que refleja ciertas coincidencias con algunos comportamientos observables con facilidad en contadores públicos, lo describen Gil-Monte y Peiró (1997), al advertir que:

[...] el de personalidad tipo A se acuñó para aludir a aquellos sujetos que se caracterizaban por manifestar impaciencia, velocidad excesiva, urgencia temporal, esfuerzo por el logro, competitividad, agresividad y hostilidad, habla y gestos bruscos y compromiso excesivo por el trabajo. Son sujetos proclives a las enfermedades coronarias y a las alteraciones cardiovasculares. (p. 73)

Este tipo de personalidad A ya había sido identificada en contadores públicos. En investigaciones realizadas en Estados Unidos se afirma que este tipo de personalidad caracteriza a buena parte de los auditores que laboran en multinacionales de auditoría, y que precisamente es su tipo de personalidad lo que lleva a este profesional a elegir este tipo de trabajos, pero sus consecuencias físicas no se pueden evadir, principalmente los riesgos de sufrir afecciones cardíacas como producto del estrés. (Fogarty, et. al., 2000; Fisher, 2001; Donnelly, et. al., 2003)

Como la experticia del autor no es la psicología, las ideas siguientes se abordan buscando una aproximación a las categorías que pudieran relacionarse con la personalidad del contador público, lo cual se hace a través del mismo proceso de búsqueda de información. Al respecto, se observa con curiosidad e interés que la personalidad tiene tipologías, rasgos, estructuras, escuelas y teorías. La aproximación se realiza sobre fundamentos de autores clásicos y contemporáneos como Hipócrates, con el tema de temperamento; Jung, con el tema del arquetipo; Freud, con la teoría psicoanalítica; Spranger, con la personalidad teórica, social, política, religiosa y artística; y escuelas de la personalidad como la del psicoanálisis, teoristas del aprendizaje, humanistas, teorías del comportamiento, y teorías de la personalidad como la individual y la social.

En relación con la "personalidad obsesivo compulsiva" propuesta por Piñuel (2004), y en la cual se enmarca al contador público, no se evidencia un soporte teórico amplio de tal categorización de personalidad. No obstante, sí se ubicó bajo la disfunción denominada "trastorno obsesivo compulsivo (TOC)" que, de hecho, ya es una manifestación de enfermedad que empeora el panorama del ejercicio del contador público. 


\section{Contex to Laiza, E.}

Gil-Monte \& Peiró (1997) se aproximan a una caracterización del tipo de profesional en la que la vulnerabilidad a "quemarse" por el trabajo o sufrir síndrome de burnout es muy alta.

Existe cierto acuerdo entre los investigadores que estudian el síndrome de burnout, al considerar que los profesionales más proclives a "quemarse" por el trabajo son sujetos empáticos, sensibles, humanos, con dedicación profesional, idealistas, altruistas, obsesivos, entusiastas y susceptibles de identificarse excesivamente con los demás. (1997, p. 73)

La caracterización del contador público propuesta por Piñuel a lo mejor no se aleja mucho de la caracterización de Gil-Monte \& Peiró, sobre el tipo de profesional proclive a sufrir semejante trastorno por el alto nivel de dedicación, laboriosidad y compromiso que, por lo menos en la teoría, se requiere en un contador público. Ahora bien, los niveles de obsesividad y compulsividad podrían ser conductas adquiridas como producto de los niveles de responsabilidad legal y social a los que se ve sometido este profesional. Esto se podría contrastar con lo planteado por Moreno (2007) al referirse al modelo modular de personalidad:

El modelo de personalidad como sistema de procesos insiste en la importancia de los procesos que generan y mantienen los perfiles de conducta y de interacción con la realidad. Más que describir la personalidad del alcohólico o del drogadicto como un perfil de rasgos, el modelo de personalidad, como sistema de procesos, insiste en los procesos que generan unas conductas y mantienen las mismas. (p. 11)

Los rasgos de workaholismo o adicción al trabajo observados en algunos contadores a la luz de esta propuesta, cobran importancia a causa de los procesos y la interacción de los mismos en función de las creencias, sentimientos y conocimiento dentro del ámbito de sus actividades profesionales, y eso se puede observar con facilidad en los contadores.

En temas relacionados con la salud mental y el trabajo es aventurado generalizar, ya que no todos los contadores desarrollan los mismos niveles y formas de estrés. Existe, por ejemplo, un esfuerzo voluntario que va más allá de lo exigido por la organización y que, al parecer, no es tan perjudicial para la salud mental, y se denomina "engagement", el cual podría considerarse como lo contrario al síndrome de burnout, y se puede estar presentando en algunos contadores públicos, lo que confirma en parte la afirmación de Piñuel sobre workaholismo (2004).

\section{Conclusiones}

El cotejo entre los antecedentes de investigaciones realizadas y la literatura especializada revisada en relación con el comportamiento y la conducta del contador público colombiano muestra grandes coincidencias entre el ejercicio de la profesión contable, el deterioro de la salud física y mental, y la caracterización tipológica de la personalidad del contable. No se puede 
sugerir que sea el común de los contadores públicos, pero estas conclusiones se presentan como una aproximación metódica al estudio del fenómeno.

Ante el panorama expuesto, el autor asume una postura crítica, y de esta forma, el tan anhelado "éxito" en el desempeño de la profesión contable trasciende dialécticamente, y se toma como punto de partida que motiva la observación metódica y sistémica del ejercicio de la profesión contable en Colombia, así como su posible efecto en la salud física y mental. Se aborda como fenómeno que se debe estudiar con rigurosidad científica, y bajo este enfoque no sólo se examine la prevalencia del estrés y el síndrome de burnout en el contador público colombiano y los derivados mecanismos de protección y afrontamiento sino también el impacto en la productividad y en la calidad de los informes contables, así como los costos objetivos y subjetivos de intervención y prevención de la salud.

Otro aspecto que se pone a discusión se relaciona con la necesidad de sincerar las competencias del contador público abordadas desde los programas de contaduría pública para insertar en estos una revelación de realidades ocultas como una alerta temprana para el futuro profesional del área contable.

\section{Referencias bibliográficas}

Almer, E. \& Kaplan, S. (2002). The effects of flexible work arrangements on stressors, burnout, and behavioral job outcomes in public accounting. Behavioral Research in Accounting, 14(1), 1-34. doi:10.2308/ bria.2002.14.1.1

Arredondo, C. \& Villavicencio, A. (2007). Estrés del contador público. Memoria XIX Congreso Internacional de Contaduría, Administración e Informática. México: UNAM.

Birnberg, J. (2000). The role of behavioral research in management accounting education in the 21st century. Issues in Accounting Education, 15(4), 713-728.

Cadogan-McClean, C. (2009). An examination of resilience: Its relationship to work stress among accountants practicing in Barbados (Ph.D.). Ann Arbor, United States. Recuperado de http://search.proquest.com/ docview/305164025/abstract/13D11CF37E37FCC4DB5/1?account $\mathrm{id}=43746$

Calvo, A. (2007). Sociología de la profesión contable en Colombia. Revista Contaduría Universidad de Antioquia, 50, 77-92.

Ciftcioglu, A. (2011). Investigating occupational commitment and turnover intention relationship with Burnout Syndrome. Business and Economics Research Journal, 2(3), 109-119. 


\section{Contexto Loaiza, E.}

Colombia, Congreso de la República . Ley 73 (1935).

Colombia, Presidencia de la República. (1956). Decreto 2373, por el cual se reglamenta la profesión de contador, y se dictan otras disposiciones. Diario oficial, 29.154. Recuperado de http://www.mineducacion.gov.co/1621/ articles-102582_archivo_pdf.pdf

Colombia, Presidencia de la República. (1971). Decreto 410, Código de Comercio de Colombia. Recuperado de www.mincit.gov.co/descargar. php?id=66161.

Colombia, Congreso de la República. (1990). Ley 43 de 1990, Por la cual se adiciona la Ley 145 de 1960, reglamentaria de la profesión de Contador Público y se dictan otras disposiciones. Diario Oficial, CXXVII (39.602). Recuperado de http://www.mineducacion.gov.co/1621/articles-104547_ archivo_pdf.pdf

Colombia, Congreso de la República. (1995). Ley 222, Por la cual se modifica el Libro II del Código de Comercio, se expide un nuevo régimen de procesos concursales y se dictan otras disposiciones. Diario Oficial, 42.156.

Donnelly, D., Quirin, J. \& O'Bryan, D. (2003). Auditor acceptance of dysfunctional audit behavior: An explanatory model using auditors' personal characteristics. Behavioral Research in Accounting, 15, 87.

Fisher, R. (2001). Role Stress, the Type A Behavior Pattern, and external auditor job satisfaction and performance. Behavioral Research in Accounting, $13,143$.

Fogarty, T., Singh, J., Rhoads, G. \& Moore, R. (2000). Antecedents and consequences of burnout in accounting: Beyond the role stress model. Behavioral Research in Accounting, 12, 31-68.

Galindo, J. (1994). Entre la exterioridad y la interioridad. Apuntes para una metodología cualitativa. ITESO. Recuperado de http://rei.iteso.mx/ handle/11117/140

Gil-Monte, P. \& Peiró, J. (1997). Desgaste psíquico en el trabajo: el síndrome de quemarse. Síntesis.

Haskins, M., Baglioni, J. \& Cooper, C. (1990). An investigation of the sources, moderators, and psychological symptoms of stress among audit seniors. Contemporary Accounting Research, 6(2), 361-385.

Hellriegel, D. \& White, G. (1973). Turnover of Professionals in Public Accounting: A Comparative Analysis. Personnel Psychology, 26(2), 239-249. 
Hendriksen, E. (1970). Accounting theory. R. D. Irwin.

Jones, A. (2007). Antecedents and consequences of life style choice in public accounting (Ph.D.). Ann Arbor, United States. Recuperado de http://search. proquest.com/docview/304705295/abstract/13CEEC2B1C649963D47/1 9? accountid $=43746$

Kalbers, L. \& Fogarty, T. (2005). Antecedents to Internal Auditor Burnout. Journal of Managerial Issues, 17(1), 101-118.

Loaiza, E. (2012). Niveles de estrés y síndrome de Burnout: Exploración en contadores públicos colombianos año 2010 (Trabajo de grado). Universidad de Antioquia, Facultad Nacional de Salud Pública, Medellín.

Loaiza, E. (2014). Modelo de costos de alcanzar el exito: Una valoración desde la exitodinámica y la incidencia en la salud del contador público colombiano. (Tesis, Doctor en Ciencias Contables). Universidad de Los Andes, Venezuela. Recuperado de http://pcc.faces.ula.ve/

Lovelace, K., Manz, C. \& Alves, J. (2007). Work stress and leadership development: The role of self-leadership, shared leadership, physical fitness and flow in managing demands and increasing job control. Human Resource Management Review, 17(4), 374-387. doi:10.1016/j. hrmr.2007.08.001

Montes, S., Soto, M. \& Valencia, S. (2006). Paradigmas en contabilidad. Cali, Colombia: Artes Gráficos Ltda. Recuperado de http://eutimiomejia.com/ textos/pdf/libro_paradigma_en_contabilidad.pdf

Montoya, J. \& Posada, I. (2007). Estudio documental sobre los factores de riesgo psicosociales presentes en las personas que laboran en las áreas administrativas. Medellín: Universidad de Antioquia, Facultad Nacional de Salud Pública.

Moreno, B. (2007). Psicología de la personalidad: procesos. Madrid: Thompson. Recuperado de http://dialnet.unirioja.es/servlet/libro?codigo=282646

Peiró, J. \& Prieto, F. (2002). La aproximación psicológica al trabajo en un entorno laboral cambiante. En Tratado de psicología del trabajo. Vol. I. La actividad laboral en su contexto. Vellehermoso, Madrid: Síntesis, S.A. Recuperado de http://www.lalibreriadelau.com/a-p20629

Phillips, J. \& De Lange, P. (2006). The effect of the introduction of Gst on accountants' job satisfaction and burnout. Australian Accounting Review, 16(3), 52-59.

Piñuel, I. (2004). Neomanagement: Jefes tóxicos y sus víctimas. 4.a ed. Madrid: Aguilar. 


\section{Contexto Loaiza, E.}

Rebele, J. \& Michaels, R. (1990). Independent auditors' role stress: Antecedent, outcome, and moderating variables. Behavioral Research in Accounting, 2,124 .

Revista Semana. (2009, junio). Se busca Bilingüe. Un estudio revela que el 30 por ciento de las empresas en el mundo tienen problemas para conseguir el talento que buscan. Identifica 10 perfiles laborales más difíciles de encontrar en el país. Recuperado de http://www.semana.com/economia/ busca-bilingue/125283-3.aspx

Senatra, P. (1980). Role conflict, role ambiguity, and organizational climate in a public accounting firm. Accounting Review, 55(4), 594-603.

Trejo, M. (2012). Fenomenología como método de investigación: Una opción para el profesional de enfermería. Enf Neurol, 11(2), 98-101.

Ussahawanitchakit, P. (2008). Building job satisfaction of certified public accountants (cpas) in Thailand: Effects of role stress through role conflict, role ambiguity, and role overloadun. Journal of the Academy of Business \& Economics, 8(2), 12-22.

Villavicencio, A. (2006). Estrés en estudiantes de Contaduría Pública. Negotia, Revista de investigación de negocios, 6(10). Recuperado de http:// www.admin.escatep.ipn.mx/Data/Sites/1/SharedFiles/pdf/investigacion/ Negotia6.pdf

Viloria, N. (2001). Epistemetodología de la Ciencia Contable. Actualidad Contable Faces, 4(4), 63-71.

\section{Para citar este artículo:}

Loaiza, E. (2014). El ejercicio de la profesión contable en Colombia. Una mirada desde el paradigma contable del comportamiento basado en la conducta y el deterioro de la salud física y mental. En-Contexto, $2,147-164$. 